Review

\title{
Molecular Communication in Nanonetworks
}

\author{
Hao Yan ${ }^{1}$, Ge Chang ${ }^{1}$, Tianhao Sun ${ }^{1}$, Yingzhan $\mathrm{Xu}^{1}$, Zhongke $\mathrm{Ma}^{1}$, Tao Zhou ${ }^{1}$, Lin $\mathrm{Lin}^{2}$ \\ ${ }^{1}$ Institute of Nano Biomedicine and Engineering, Shanghai Engineering Research Center for Intelligent Diagnosis and Therapy \\ Instrument, Department of Instrument Science and Engineering, School of Electronic Information and Electrical Engineering; \\ National Center for Translational Medicine, Collaborative Innovational Center for System Biology, Shanghai Jiao Tong University, \\ Shanghai 200240, China. \\ ${ }^{2}$ College of Electronics and Information Engineering, Tongji University, Shanghai 201804, China.
}

\Corresponding authors. E-mail: fxlinlin@tongji.edu.cn; yan_hao@sjtu.edu.cn

Received: Dec. 4, 2016; Accepted: Dec. 7, 2016; Published: Dec. 20, 2016.

Citation: Hao Yan, Ge Chang, Tianhao Sun, Yingzhan Xu, Zhongke Ma, Tao Zhou, and Lin Lin. Molecular Communication in Nanonetworks. Nano Biomed. Eng., 2016, 8(4): 274-287.

DOI: $10.5101 /$ nbe.v8i4.p274-287.

\begin{abstract}
With the development of nanotechnology, bioengineering and biology, it is envisioned that biological nanomachines may flourish in assorted valuable applications considering their unique characteristics including energy efficiency, bio-compatibility and extremely small scale. However, current biological nanomachines are only able to perform simple tasks at nano-level. Therefore, nanonetworks which interconnect bio-nanomachines into a network have been proposed to overcome the limitations of individual biological nanomachine. Among the possible communication schemes for nanonetworks, modern electromagnetic communication techniques are not good solutions due to the limitation of antenna size. Inspired by nature, one promising candidate is molecular communication proposed from the perspective of communication and computer engineering. Integrated with the knowledge from communication and computer engineering, molecular communication enables biological nanomachines to interface with other biological nanomachines and existing biological systems. Their interconnections form a bio-nanonetwork which is capable to provide functions that individual nanomachines cannot accomplish. In this paper, we introduce the state-of-the-art progress in the emerging field of molecular communication. The framework, design and engineering of components and theoretical modeling of molecular communication are discussed. The research challenges and opportunities are also talked about to inspire future researches of more feasible molecular communication systems.
\end{abstract}

Keywords: Biological nanomachines; Molecular Communication; Nanonetworks

\section{Introduction}

Looking back in 1950 s, the possibilities of functional and powerful nano-devices were noted in Richard Feyman's famous speech "There's Plenty of Room at the Bottom". After more than 60 years, with the development of biology, nanotechnology, and material science, various biological nanomachines (bio-nanomachines) which are capable to interact with molecules and cells in nanometer to micrometer level have been engineered. Bio-nanomachines are referred to nano-to-micro scale functional devices made up of biological materials and can perform simple chemical tasks at nanoscale [1]. Examples of bio-nanomachines include genetically engineered cells, artificial protocells, bio-silicon hybrid devices [2] and nanoscale 
molecular complexes such as protein motors [3].

The advantages of bio-nanomachines include energy efficiency, bio-compatibility and extremely small scale. What is more important is that bio-nanomachines are capable to interface with other bio-nanomachines and bio-system. Due to the above advantages, bionanomachines have the potential to deal with problems beyond the reach of traditional application, such as drug delivery and bio-hybrid implants in bio-medical applications, biochemical sensors for pollution control in environmental applications and etc.

Though bio-nanomachines have many advantages, current bio-nanomachines are only capable of performing simple tasks because of their limited size and structure. To overcome this limitation, nanonetworks which interconnect bio-nanomachines and integrate them into a network have been proposed. Among the possible communication schemes for bionanonetworks, modern electromagnetic communication techniques are not good solutions due to the limitation of antenna size. On the other hand, molecular communication which is universally distributed in plants and animals has proven to be energy efficient as well as reliable. Inspired by nature and bionanomachines' advantages, molecular communication (MC) [4] is proposed as an ideal communication paradigm for bio-nanonetworks from the prospective of communication and computer engineering. Researchers from communication and computer engineering have made contributions to $\mathrm{MC}$ in nanonetworks. An initial attempt to establish MC network was conducted by Suda in 2005 [4]. Assorted MC models were also proposed in [5-10]. Related experiments were pioneered in $[5,11]$. Current progress was included in surveys which presented the general micro-scale MC and characteristics of MC [1, 12-19].

Nevertheless, there are still many difficulties to overcome before feasible applications. First of all, current MC system models, which are the key to a proper communication process, are based on many unrealistic assumptions. They are too simple and idealized. Some important factors for reliable applications are not well addressed, such as the power source to support MC and synchronization between bio-nanomachines and etc. One of the biggest challenges is experiment validation of bionanonetworks. This needs further collaboration of multiple disciplines including bioengineering, biology, chemistry and communication engineering. The main purpose of this paper is to introduce computer and communication assisted $\mathrm{MC}$ to bio-engineering domain to advance collaborations and to promote development of MC.

In this paper, the advancement of $\mathrm{MC}$ in nanonetworks is reviewed. First, an overview of MC is provided. Its architecture, potential power sources, general characteristics and potential applications are introduced. Second, approaches for design and engineering the components of MC are discussed. The characteristics of bio-prototypes of bio-nanomachines discussed here could be further considered in the actual MC or for more realistic model of MC. And, different $\mathrm{MC}$ theoretical models for different situations are presented. Models also include general model and specific models. These models can be used to facilitate MC design and analysis. Finally, possible future works are discussed. These works aim to solve the key research issues and promote the advancement of $\mathrm{MC}$.

This paper is organized as follows. In section two, we introduce the MC including the architecture, characteristics and potential applications. The power sources of the MC are discussed separately. Section three provides a summary of bio-prototypes that could be used in MC. And bio-nanotechnology approaches to engineer and design the components for $\mathrm{MC}$ are discussed. The approaches to mitigate the interference are also included. In section four, we categorize the MC channel models into diffusion models and other emerging models based on its propagation ways. Qualities of some frequently used models are discussed correspondingly. Section five considers the challenges of the potential MC systems and possible future works are talked about. Finally, section six concludes this paper.

\section{Overview of Molecular commu- nication}

The process of a MC system can be simplified as three main parts: generation and release of information particles, propagation of information particles in the environment, detection of information particles. We can abstract the process above into an architecture to identify the key components of MC. As shown in Fig. 1, the MC architecture contains three main modules (transmitter, channel and receiver) and their submodules. The transmitter generates information particles, and then releases them to the channel. The 


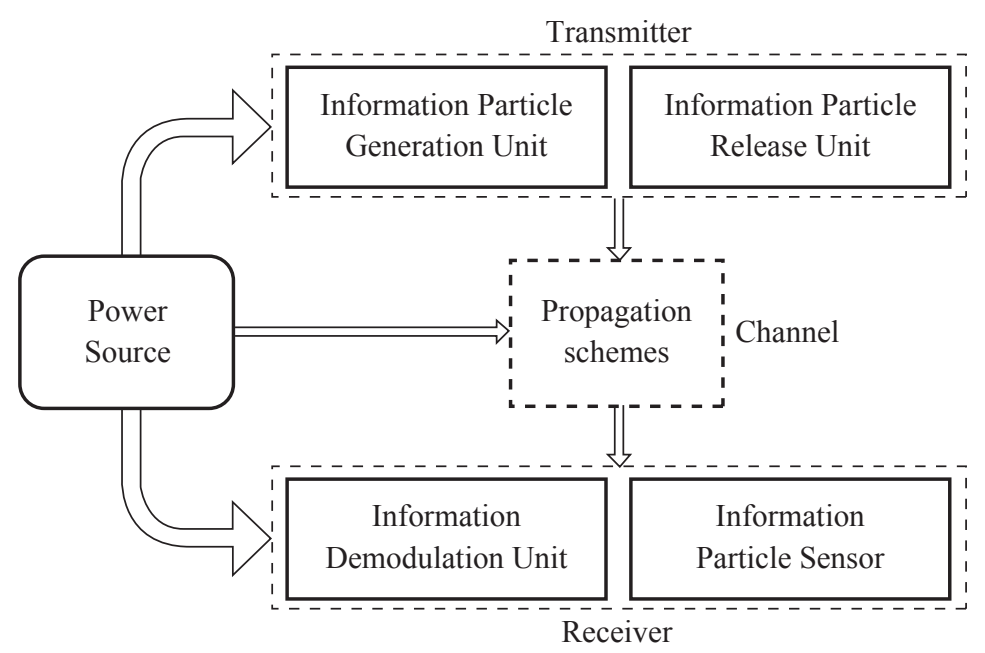

Fig. 1 Key components of molecular communication.

channel acts as the environment where information particles propagate by specific method, including diffusion, active transport, etc. It cannot be ignored that noise exists in the channel and influences the accuracy of information communication. Therefore, it is a big challenge for us to overcome the noise and realize a reliable MC. Finally, the receiver captures the information particles and utilizes their features to obtain the intended information from the transmitter.

In this section, based on the architecture discussed above, we overview the three main modules at first. Especially, the power source of the system is introduced separately. We also discuss its general characteristics and potential application to give us a comprehensive view of MC and its promising future.

\section{Molecular communication architecture}

\section{Transmitter}

The transmitter of MC is required to generate and release information particles, which is corresponding to the Information Particle Generation Unit and Information Particle Release Unit in Fig. 1 (Power Source will be introduced in the following part). The main function of a transmitter is to send different information, which is achievable easily in modern radio-based communication systems. But in $\mathrm{MC}$, the information carrier is the particle so that existing theory about electromagnetic wave cannot support the transmitting process. So new approaches have been proposed for sending information by utilizing different properties of information particles. The receiver can then decode the information particles to get the intended information. The properties used to modulate are shown below:
- Concentration of Particles.

- Type of Particles.

- Three-dimensional-structure of Particles.

- Release Time of Particles.

In modern radio-based communication systems, symbol bit is the fundamental unit of information and the propagation process of information can be regarded as transferring binary data (0 and 1$)$. This performs similarly in MC. Resembling in Fig. 2, different binary data ( 0 or 1 ) is obtained by modulating on concentration of Particles, type of Particles, threedimensional-structure of Particles [20] and release time of Particles. For example, for the graph in the left top, three molecules in a column represent bit 0 , while one molecule represents bit 1 .

In addition, the release mechanism of information particles is a significant issue of transmitters. There are many natural methods to release chemical particles intracellularly or intercellularly, including budding vesicles from a cell, opening molecular gates, etc. [16]. Therefore, the release mechanism of information particles in MC can work in the similar ways.

It is worth mentioning that because of the degradation of particles, the transmitter needs to release a large number of particles to make the communication process more stable. The properties of information particles should also be considered, which need to be chemically robust against the noise from the environment.

\section{Channel propagation}

The channel in MC system is the environment where the information particles propagate from transmitters 


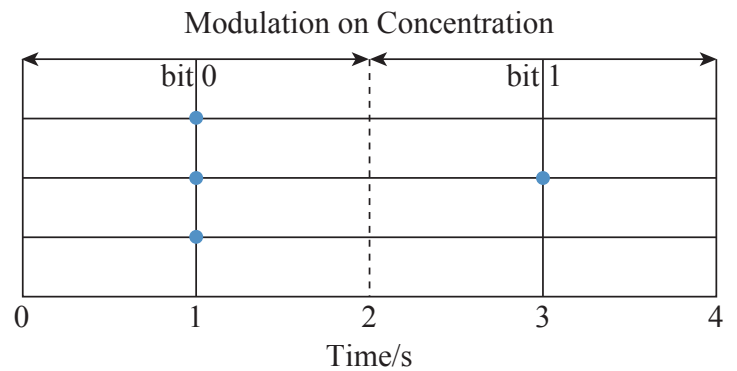

Modulation on Three-Dimensional-Structure

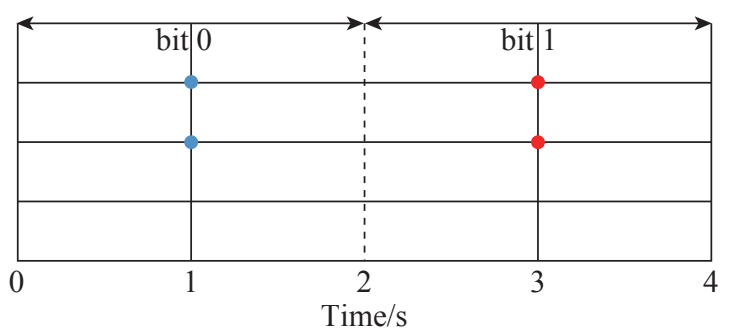

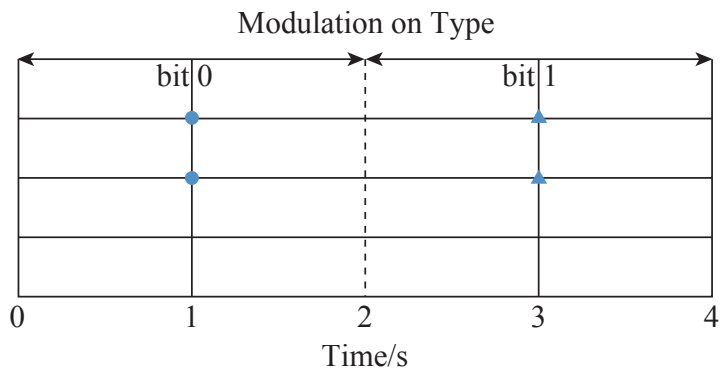

Modulation on Release Time

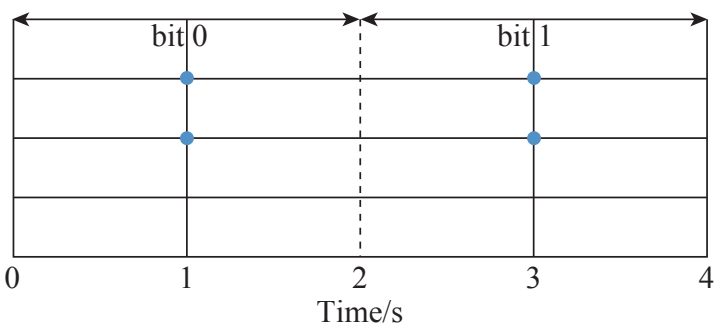

Fig. 2 Modulation techniques in molecular communication.

to receivers. For example, the vessel provides the environment for erythrocyte, protein and many other materials to transport between certain tissues at a molecular level. In Table 1, schemes which have been proposed for the propagation of information particles in the channel are listed. Among them, the diffusion based propagation is the most frequently used scheme in current studies.

Table 1 Propagation Schemes in Molecular Communication

\begin{tabular}{cc}
\hline Progation Schemes & Based on \\
\hline Free Diffusion & Diffusion \\
Diffusion with First Hitting & Diffusion \\
Flow Assisted Diffusion & Diffusion and Flow \\
Motor Protein over & Motor Protein \\
Microtubule Tracks & Bacteria Motility \\
Bacteria Assisted & Diffusion and Gates \\
Gap Junction & Diffusion and Enzyme \\
Neurochemical &
\end{tabular}

For instance, free diffusion in Table 1 occurs when gaseous or aqueous molecules move without any other force. The movement of the molecules obeys the Brownian rule. The mathematical modeling will be discussed in later section. Different schemes adapt to different conditions of environment, and all of them are important for practical applications.

To have a further study of the channel in $\mathrm{MC}$, we need to set up the channel model according to the propagation schemes we choose. Frequently used models include diffusion based model, flow assisted diffusion model, etc.

\section{Receiver}

In nature, one way that biological cells capture signals is to use protein structures, which can bind to specific ligand structures [21]. This mechanism can be used in MC to design Information Particle Sensor of a receiver to detect and receive information particles [22, 23]. Another choice of receiver is to use a permeable surface structure (e.g., plasma-membrane) or channels for specific molecules (e.g., ion channels) [16].

When receiving the information particles, the receiver comes to a process called demodulation. The Information Demodulation Unit of the receiver demodulates the intended information from the properties of information particles, which include the concentration, the type, the three-dimensionalstructure and the release time of particles as mentioned in Transmitter. With receiver acting like a cell, the demodulation process works via certain chemical reactions with the information particles.

\section{Power source}

The transmitter, propagation in channel and the receiver all require adequate power to work regularly. The ways to harvest power for MC [24] can be summarized into three categories as follows:

- Getting power present in the environment. Diffusion based propagation utilizes the thermal energy from the channel. This process can work without any external power source.

- Getting power through chemical reaction from the 
environment. For example, in a biological cell, chemical reactions are used to generate power for motion to propagate chemical particles, such as the utilization of adenosine-triphosphate (ATP) hydrolysis [25].

- Getting power with an external power source [26, 27]. For instance, we have mentioned a propagation scheme called Flow Assisted Diffusion, which utilizes a flow in the channel to provide the power for particles' movement in a certain direction while diffusing. The flow can be regarded as a kind of external power sources.

It should be pointed out that the energy efficiency is high when the MC process harvests power present in the environment or through the reaction in the environment. The diffusion-based propagation only needs the thermal energy in the channel without any external power source so that it is an energy efficient process. In addition, take myosin molecular motors as an example. They work via converting chemical energy like ATP with approximately 100 percent efficiency.

\section{General characteristics}

Compared with traditional radio-based communication, MC shows many differences such as the information carrier, the information propagation speed, and the medium [28]. As a result, the general characteristics of MC are much different from radiobased communication. Important characteristics of MC are introduced as follows:

- Chemical particles as information carriers. In MC, information is modulated on the properties of chemical particles, which include the type, the concentration, the release time of particles, etc. A good example to describe this is neurotransmitter, a kind of chemical materials existing in synapse and propagating information from our brain to muscles all over the body. In this case, the information from the brain is modulated on the type of particles and other kinds of particles cannot convey the intended information to muscles. In addition, because of the chemical properties of these particles, the modulation and demodulation process can be performed with a series of chemical reactions.

Compatibility for biological systems. The communication mechanism in MC is similar to the one used in biological systems, both of which implement communication process via the movement of chemical particles as imformation carrier and the chemical reactions. Therefore, it is possible that the bio-nanomachines could directly communicate with components of natural biological system. It also shows a potential application that MC systems with bio-nanomachines can be implanted inside a human body to do medical treatment.

- Slow propagation speed and high loss rate. Compared with electromagnetic wave, the propagation of information particles in MC is much slower and the propagation distance is much shorter. For free diffusion, the propagation is even limited to micrometer range. Due to the diffusion property of the propagation, the particle movement is unpredictable and the distribution range of the arriving time is large. Therefore, the loss rate is high, and those arriving at the receiver only account for a small percentage.

\section{Potential applications}

With the recent advancements in nanotechnology, the potential applications of MC systems have become increasingly feasible. The application areas include medical field [29], environment [30], industry, military, etc. Here we introduce some of important application areas as below:

\section{Medical applications}

Nowadays, the medical field has become the main force for engineering MC.

A potential application in medical field is the artificial immune system [31]. In this application, bionanomachines are implanted into the human body and communicate with each other via the MC. One device can only perform simple tasks, while complex work needs the cooperation among the devices. This process is similar to the immune system in human body, in which T-cells and B-cells are required to work together.

Drug delivery [32] is another promising application. Drug delivery systems perform accurate transport of drugs to specific locations. Bio-nanomachines are implanted inside the body to detect the molecular signal from specific targets and precisely position their accurate locations for drug delivery. This application reduces the side-effects on locations which are not targets.

Other potential applications include the detection of brain aneurysm, transport of molecular payloads between tissues, lab-on-a-chip devices [33], etc. 


\section{Environmental applications}

Nanomachines and MC systems have the potential to help environmental monitoring and protection. Feasible applications are as listed:

- Pollution control. The toxic or radioactive agents in the environment can be detected by nanomachines. And then the devices tag the pollution source with specific molecules as well as amplify the signal of pollution source to guide other devices to degrade the materials in the polluted area.

- Bio-degradation assistance.

- Biodiversity control.

\section{Industrial applications}

The MC system can be used in quality control of food and water, since the type or the concentration of molecules in the goods can be a judge standard of the quality. Also, it can work on the manufacturing process of intelligent functionalized materials.

Other aspects like military applications (biological and chemical defenses, nano-functionalized equipment) are also popular in the society today.

\section{Design and Engineering of Components for Molecular Comm- unication}

The MC systems require interdisciplinary cooperation for their design and engineering, such as biology, chemistry, and nanotechnology fields. And the systems are realized from biological mechanisms and materials. As shown in the previous section, many components for MC can be found the prototypes in biosystems or get implementation through biotechnology, including the transmitter and receiver, information particles, guide and transport molecules, power source molecules. There are errors in the process of propagation and detection, like ISI (Inter Symbol Interference), we have to consider the component for error correction. In this section, we summarize research results for engineering components that are applicable to developing MC systems.

\section{Transmitter and receiver}

Transmitter and receiver require some special functionalities for effective communication. At the transmitter, capabilities are required for generation or storage of information particles. There may also be a need for a mechanism that controls the release of information particles, a processing unit that controls the different processes within the transmitter. At the receiver, there must be a sensor, or a receptor, or a detector that can measure some arrival property of the received information particles. If coding techniques are used there may also be a need for a central processing unit for decoding and deciphering the received signal.

In $\mathrm{MC}$, one can synthesize the transmitter and receiver in two basic ways: shrinking existing electronic components to nano scale, and designing and manufacture by bio nanotechnology. Dislike the difficulty and high cost for shrinking components, research results show that adopting bio technology can help us get more efficient transmitter and receiver. And Two basic approaches for engineering bionanomachines with functionality for communication are modification of existing biological cells and simplification of cell-like structures using biological material [34].

\section{Modification of existing cells}

The first approach for engineering bio-nanomachines was proposed by synthetic biology [35-37]. To perform a sending function, a biological cell with a modified a metabolic pathway is capable to synthesize and release specific signal molecules. Furthermore, another work considered both the transmitter and receiver are genetically modified cells and mobile [38]. And an information transport mechanism for the two genetically modified cells was designed. Microbial colonies are also one of the prominent transmitter and receiver entities in MC [39]. In this scheme, the transmitter and receiver are made from gene transfected cells by the transfection of genetically modified bacteria.

Many other functions can be introduced into biological cells with the help of synthetic biology, such as logic gates, toggle switches, and oscillators. These functions can increase the complexity of transmitting and receiving processes. An example of functions which could be embedded in bio-nanomachines is oscillators to generate the clock for MC.

\section{Creating artificial cells}

To engineer transmitter and receiver bionanomachines, simplified cell-like structures using biological materials is one solution. One example is utilization of a liposome that separates the environment 
and functional particles. functionality can be added into liposome as necessary. The liposome is a structure wrapped by lipid bilayer which is similar to the membrane that encloses a cell [2]. In [40, 41], transmitter and receiver bio-nanomachines were synthesized from vesicles embedded with photoresponsive molecular switches. These devices were photo-controlled to transmit or react to chemical signals, and thus demonstrated the potential of this approach for creating bio-nanomachines.

\section{Information particles}

Information particles propagate from a transmitter bio-nanomachine, carry information through the channel and finally detected by a receiver bionanomachine in the environment. The choice for the information particles is important with the requirements of chemically stable and robust against environmental noise and interference from other molecules. And their size and structure may affect the information transfer efficiency. There have been some examples of information particles used in nature by biological systems as follows:

\section{Hormones}

Hormones are some efficient active substances in body as a messenger to convey information to regulate the physiological processes of the body, secreted by the endocrine glands or endocrine cells [1].

\section{Pheromones}

Pheromones (also named external hormones) play a key role in the communication between the individuals. The receiver for the particles is olfactory organ which reacts with the pheromones to lead a direction to another individual [2].

\section{Neurotransmitter}

Acetylcholine (Ach) is one of the most widely used neurotransmitters. Ach performs at the information transport in Neuromuscular junction [3]. Ach is released by the presynaptic terminal which is a special structure of the junction that performs as the transmitter, and is detected by the postsynaptic terminals which performs as the receiver. The receiver control the strain and relaxation of muscle according to the concentration of the received Ach.

\section{Deoxyribonucleic Acid (DNA) and Ribonuccleic Acid (RNA)}

As the necessary genetic materials and message molecules in body, DNA and RNA chains are both made of 4 kinds of Deoxyribonucleic acids or Ribonuccleic acids with different bases (For DNA is adenine, thymine, guanine, cytosine. And for RNA is adenine, uracil, guanine, cytosine). Different bases combinations encode different messages [4].

Besides the previous examples, in biological systems, information molecules can also be synthesized for specific purposes using bio-chemicial technology, for example, using nanoparticles to target particular tissue types in drug delivery [42].

\section{Guide and transport molecules}

Guide and transport molecules provide reliable mechanisms of directing information molecules toward target in propagation. Numerous examples in biological systems can be used to design and engineer guide and transport molecules as below:

\section{Gap junction channels}

Gap junction is an intercellular communication pathway [43-45]. In the gap between the connecting points of two cells, a small molecule is allowed to flow directly from one cell to another through the gap. These channels regulate the propagation of information molecules between cells through gap junctions. Thus receiver bio-nanomachines can be targeted by selecting where gap junction channels lead.

\section{Molecular motors and microtubules}

Microtubules self-organize into a network and motor proteins actively transport information molecules along tracks of the microtubules [5]. The specific patterns of microtubule filaments can be designed to form a pathway to direct molecular motors to target locations of the network, just like the way that trains and tracks work.

\section{Self-propelling organisms}

Self-propelling organisms like bacteria can function as transport molecule [46]. For example, bacteria can be guided by the concentration gradient of attractant molecules to move toward a receiver in the environment. The attractant molecules, generated by the receiver, function as guide molecules to influence the direction in which the transport molecules move to the receiver through the environment.

\section{DNA or RNA}

As shown in the previous subsection, DNA 
and RNA chains are both made of 4 kinds of Deoxyribonucleic acids or Ribonuccleic acids with different bases. Above all, these Pairing of bases is only possible in a specific way, for DNA is A(adenine) with $\mathrm{T}$ (thymine), $\mathrm{G}$ (guanine) with $\mathrm{C}$ (cytosine), and for RNA is A(adenine) with $\mathrm{U}$ (uracil), $\mathrm{G}$ (guanine) with $\mathrm{C}$ (cytosine). A DNA segment can function as a transport molecule with information molecules loading on it. With the fixed rule of base pairing, the transport DNA segment binds with a specific site of the target DNA segment in the receiver and therefore transfers the information molecules to the receiver.

\section{Power source molecules}

In $\mathrm{MC}$, the processes of transmitting, receiving and propagation may require power [24]. Sometimes the power is already present in the environment. Sometimes it is harvested from the environment through chemical or electrical reactions. There are some sophisticated energy supplying mechanisms relying on molecules in nature that we can used in MC. For instance, transmitters and receivers made from synthetic cells run on adenosine triphosphate (ATP) molecules which is already present in the environment [47].

\section{Error correction components}

When the information particles were detected by the receiver, they will not immediately disappear and exist for a period of time at the receiver side. Considering the pulse signal moduled by the number of information particles, the existing particles of the last pulse will affect the current information received, which is called ISI (Inter Symbol Interference). To correct the ISI, numerous methods in bio-system can be used as follows:

\section{Enzymes}

The enzyme is the initiator of the chemical reaction in the organism. For instance [6], at the receiver area of neuromuscular junction, there are some acetylcholinesterases (enzyme for Ach) breaking down the Ach molecules when they are accumulated to a certain concentration to avoid the cause of ISI.

\section{Changes in $\mathrm{PH}$ in the environment}

Comparing to electronic components, molecules are more sensitive to the degree of $\mathrm{PH}$ in the environment .The changes of $\mathrm{PH}$ in the environment may cause hydrolysis reaction, dehydration condensation reaction and other chemical reaction which degrade information molecules at receiver.

\section{Binding and absorbing}

In nature, some receptors remove the information molecules from the environment through binding and absorbing. These mechanisms can be adopted in MC to degrade the unwanted information particles [48].

\section{Theoretical Modeling of Molecular Communication}

Theoretical models are the mathematical foundation of MC. They serve as the powerful tool in designing and analyzing new mechanisms as well as improving quality of MC. A number of channel models have been proposed based on different propagation schemes (e.g., diffusion, protein motor, micro-tubule Filament, and bacterium assisted). From the perspective of current $\mathrm{MC}$ research tendency. We categorize current theoretical models work into diffusion models and some other promising models such as active transport model. The model's characteristics of pure diffusion, flow-assisted diffusion and diffusion with amplifier are discussed in the first subsection $[16,49]$. The quality of different theoretical models of MC is evaluated in terms of some pivotal factors such as channel capacity, average latency and loss rate.

\section{Diffusion based model}

Diffusion, also known as Brownian motion, is used to describe the particle's random walk during which it collides with other particles [17]. Propagation from the transmitter to the receiver can be achieved by diffusion without using external energy. Shown in Fig. 3, Transmitter is usually abstracted as a point and receiver may contain various receptors to decode different information molecules. Shown in Fig. 3, Transmitter is usually abstracted as a point and receiver may contain various receptors to decode different information molecules. Generic diffusion equation is used to describe a large number of particles' diffusion [17]:

$$
\frac{\partial p\left(d, t \mid d_{0}\right)}{\partial t}=D \nabla^{2} p\left(d, t \mid d_{0}\right)
$$

where $D$ is the diffusion coefficient, $p\left(d, \mid t_{0}\right)$ is the concentration function of current location $d$ and time $t$ and $d_{0}$ is the original location. Variable $d$ is usually represented in a Cartesian coordinate. More specific 


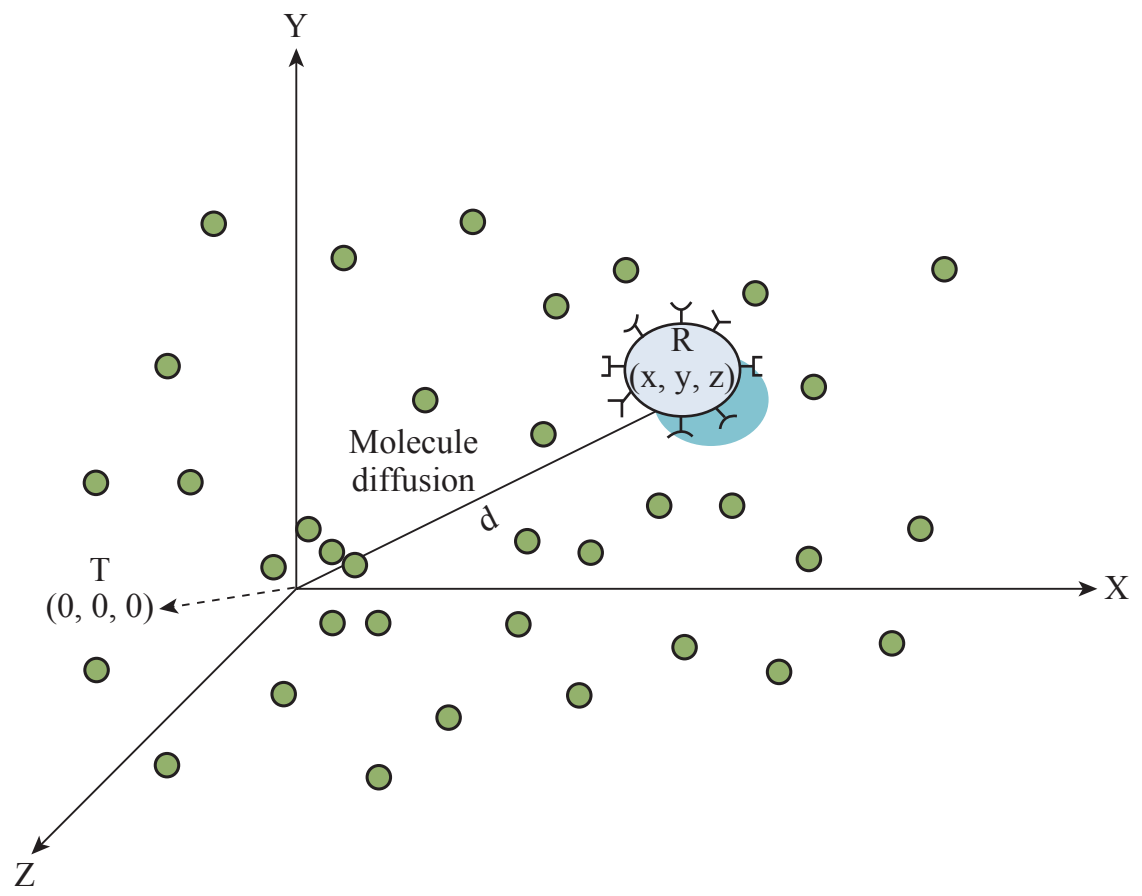

Fig. 3 Diffusion process in nolecular communication.

diffusion models are discussed in the following based on this generic diffusion equation.

\section{Pure diffusion-based model}

Pure diffusion-based model is the most fundamental MC channel model in literature as it doesn't require any additional propagation mechanism. Universally distributed in nature, this kind of model is mostly found in biology. One example is neurotransmitter molecules Acetylcholine (ACh) used to convey motor action from nerve cell to neuromuscular junction [50]. DNA binding propagated to the binding site is another example of this model [49].

Different initial conditions may be set to solve the diffusion equation of Eq. (1). $M_{0}$ molecules are assumed to be released from a point source suddenly at time $t=0$ [49]. The solution of Eq. (1) with the above initial condition for three-dimensional diffusion is:

$c(d, t)=M_{0}(4 \pi D t)^{-\frac{3}{2}} e^{\frac{-d^{2}}{4 D t}}$

The lower bound on channel capacity is given in [12] a closed-form expression. This article demonstrated that the bound was linear to the transmission's signal bandwidth positively. Shown in [16], average latency for the receiver is infinite when there is no bound for $\mathrm{d}$ and so is the jitter in this situation. The average latency, however, is finite when the probability mass function and time $\mathrm{t}$ are obtained between [0, $d]$ [49].
The average Latency is calculated as $d^{2} / 2 D$ and jitter is $\sum_{i=0}^{\infty}\left(i \Delta-\left(d^{2} / 2 D\right){ }^{2} p(i \Delta)\right.$

where $p(i \Delta)$ is the probability mass function and $\Delta$ is the time step length in simulation. In this case, loss rate is given as $1-\sum_{i=0}^{T} p(i \Delta)$ where the monitoring duration of receiver nanomachine is $\mathrm{T} * *$ [F 2016].

\section{Flow assisted diffusion model}

Pure diffusion is a very slow process to propagate information molecules in terms of macro-scales. Introducing flow is an effective method to speed up pure diffusion mechanism. Examples of this kind of propagation are found in blood vessel which is adopted as the flow to propagate nano-medicine to reach target cells [51].

The advection-diffusion equation is used to describe statistical movements of particles in this situation.

$\frac{\partial p\left(d, t \mid d_{0}\right)}{\partial t}+\nabla\left(v p\left(d, t \mid d_{0}\right)\right)=D \nabla^{2} p\left(d, t \mid d_{0}\right)$

where $v$ is the velocity vector. The pdf of the hitting time is given in [52] as shown in Eq. (4) where $M_{0}$ is the molecule number released by transmitter each time.

$f(t)=\frac{M_{0}}{\sqrt{4 \pi D t^{3}}} e^{\frac{-(v t-d)^{2}}{4 D t}}$ 
Latency is calculated by replacing $d^{2}$ with $(d-v t)^{2}$ in the probability mass function [17]. Average latency is $d / v$ and it will decrease in proportion to $1 / v$. Jitter is $D d / 2 v^{3}$ and it decreases as $\mathrm{v}$ increases. All the conclusions are based on the assumption that velocity of medium $\mathrm{v}$ is greater than zero. Loss rate can also be obtained by substituting $d^{2}$ with $(d-v t)^{2}$ in the probability mass function [16].

\section{Diffusion with amplifier}

Information molecules may react with other molecules or chemicals in the channel. Diffusion with amplifier helps improve the reliability and efficiency of the MC. The amplifiers in the channel react with information molecules and then make copy with them. The number of information molecules will increase with respect to propagation. Proteins can be adopted to amplify calcium ions or some other molecules for amplifier diffusion [53]. As the increase of deployed amplifiers number, latency decreases correspondingly. An increase in the number of released molecules by amplifiers each time also decreases latency [16].

\section{Other models}

Aside from diffusion propagation, we also survey some other promising propagation schemes. Bacteriabased propagation is an effective active transportation method. Information may be encoded in DNA strands and then transported by flagellated bacteria [46]. Attractant and repellent molecules are sometimes used to drive bacteria to the receiver [54]. Step angle is the key parameter in the rotational diffusion model [49] which affects the mobility pattern bacteria. With the attractant and repellent molecules, step angle may be redefined as:

$\Psi=\Psi_{0}+\Psi_{R}+\Psi_{A}$

where $\Psi_{0}$ is the original step angle and the effects from the attractant and repellent molecules is induced by angle $\Psi_{R}$ and $\Psi_{A}$.

Molecule motor based active transport is also promising in MC. Kinesin with stationary microtubule and microtube filaments gliding over kinesin-covered substrate are two feasible methods to perform active transport. This kind of propagation has a widely use in the lab-on-chip applications. Channel model of kinsein moving over microtube has been proposed in [55]. The trail of the motor protein can be modeled in onedimensional system with attached state in Eq. (6):

$l_{i}=l_{i-1}+v_{\text {avg }} \Delta t$ where $l_{i}$ is the location of the motor on the ith simulation step and $v_{\text {avg }}$ denotes the average velocity. A mathematical model of microtuble filaments is also presented in [56]. Step size $\Delta r_{i}$ and the Step angle change $\Delta \theta_{i}$ are the key parameters and they could be obtained through Gaussian distribution in Eq. (7) and (8) where $\Delta t$ is the step time and $L_{p}$ is the persistence length of the microtube's trajectory.

$\Delta r_{i} \sim N\left(v_{a v g} \Delta t, 2 D \Delta t\right)$

$\Delta \theta_{i} \sim N\left(0, v_{a v g} \Delta t / L_{p}\right)$

Gap junction channel is a scheme where the transmitter and receiver are attached to each other with the same lattice structures or cells. Calcium ions might be the information molecules travelling on these lattices This process is called intercellular calcium wave (ICW). The channel model was considered in [57] as well as the proper symbol rate. Channel capacity is also discussed in [58].

\section{Possible Future Works}

In this part, important research issues in current MC and possible future works are discussed.

\section{Experiment investigation}

Currently, one of the biggest challenges in MC field is experiment validation of MC in nanonetworks. The theoretical models need to be evaluated by experiments. Important parameters in models need to be justified by experiments. The reliability and effectiveness of various proposed MC schemes need to be tested by actual bio-nanonetworks with experiment.

The strong demand of experiment investigation is without doubt. Few experiments have been performed. The challenge of experiment lies in the multidisciplinary nature of the field. The theoretical models in MC are being developed by researcher from communication engineering field. To validate these models and apply them in envisioned applications requires wet lab experimentation. However, researchers from communication engineering usually do not have expertise of and access to wet labs. Therefore, experiments can only be performed by collaboration of researchers from communication and computer engineering, bio-engineering, biology and chemistry.

One possible convenient solution is utilization of the off-the-shelf technologies to develop the experimental 
platforms. We could expect that the most valuable new contributions to $\mathrm{MC}$ field would be the validation of the proposed models by experiments through collaboration of multi-disciplines.

Below are some experimental platforms that if developed, would have significant effect on the advancement of the field.

- MC in aqueous environments in micro-scales:

Although experimental platforms over free-air at macro-scales exist [59], there are no MC testbeds for liquid environments, especially at micro-scales. However, many expected applications are at microscales in aqueous environment. Developing such experiment systems at micro-scales can be very significant for the advancement of MC.

- Demonstration of MC for synthetic biology and nanotechnology:

Modeling and simulation are the current ways of $\mathrm{MC}$ investigation. However, the main interests of MC are in applications of systems biology and nanotechnology. Therefore, experimental systems that demonstrate MC between synthetic biological devices or $\mathrm{MC}$ in nanotechnology are tremendous valuable.

- Structure of molecules or particles as information carriers:

A large amount of information can be encoded in the structure of molecules (e.g. DNA molecules), which provide a high data rate. However, the techniques for rapid generation of different molecular structures are not available and need to be developed.

\section{Propagation models}

Various channel models have been proposed. However, they are too simplistic. For example, many works on diffusion-based propagation assume infinite boundary conditions and assume transmitter as a point source with infinite small size, which are not the case for many applications. For examples, blood vessel environment is a finite boundary condition. Therefore, more realistic and accurate models are needed.

What is more important is that current proposed models are only verified with simulations. This is not sufficient. The developed models should be validated by experiments.

Current models focus on the channel between fixed transmitter and receiver. Channel models of mobile nanomachines are seldom considered. The related investigations are very limited and not sufficient. However mobile MC is an important issue. For instance, in drug delivery, bionanomachines are mobile and move dynamically to track the target. Current models fail to accurately express such mobile MC.

Most of current works focus on diffusion-based propagation. There are also other propagation approaches in MC including bacteria-based propagation, motor protein-based propagation and extracellular matrix-based propagation. Very few works have considered the channels of these propagation ways. More investigation will be necessary.

\section{Modulation process}

Many modulation schemes have been proposed for MC channels $[19,60]$. However, there are a lot of simplifying assumptions in the schemes. And the proposed schemes only are evaluated by simulations. Therefore, one of the biggest challenges is to validate these modulation schemes by experiments.

Synchronization is an open issue. Most of the proposed modulation schemes for MC assume that the transmitter and the receiver are synchronized. However, how they are synchronized is unknown. Some previous works have proposed solutions of this problem [61-63]. But further investigations are still needed.

Parameter estimation is also an important issue. In $\mathrm{MC}$, channel parameters are required at the transmitter or receiver sometimes. Typical channel parameter could be distance between the transmitter and the receiver, diffusion coefficient of medium and etc. [64, 65].

In MC channel, ISI is the main interference source and has a significant impact on the performance of modulation [66]. Therefore, effective ISI mitigation techniques are worthy of attention.

\section{Conclusions}

MC is a multidisciplinary field at the intersection of electronic communication and computer engineering, biotechnology, biology, chemistry, and etc. Although $\mathrm{MC}$ is still in its infancy, much advancement has been achieved in developing theoretical models for MC in the communication engineering society. But we are still facing many challenges to be solved. At the same time, the fields of biotechnology, synthetic biology, and nanotechnology progress rapidly. Primitive devices with limited capabilities have been developed and could be applied into implementation. 
We are at a critical stage in the development of $\mathrm{MC}$ to welcome some important applications on the horizon. This relies on the effective collaboration of the scientists from all the related disciplines. The goal of this survey is to present a communication theoretic formulation of $\mathrm{MC}$ that may not be familiar to researchers from biotechnology, biology, and chemistry. We hope that this survey could motivate more collaboration between researchers from these disciplines and fosters experimental works soon in the MC field.

\section{Acknowledgments}

The research leading to these results has received funding from the National Key Basic Research Program (973 Project) (No.2015CB931802), National Natural Science Foundation of China (Grant No. 61502295 and Grant No. 61405111), the Scientific research Foundation for the Returned Overseas Chinese Scholars through the State Education Ministry (Grant No. 20141685), the Shanghai Sailing Program through the Science and Technology Commission of Shanghai Municipality (Grant No. 14YF1408700), and the College and University Young Teachers' Training Program through the Shanghai Municipal Education Commission.

\section{References}

[1] I.F. Akyildiz, J.M. Jornet, and M. Pierobon, Nanonetworks: A new frontier in communications. Communications of the ACM, 2011. 54(11): 84-89.

[2] M.J. Doktycz, M.L. Simpson, Nano-enabled synthetic biology. Molecular Systems Biology, 2007. 3: 125.

[3] A. Goel, V. Vogel, Harnessing biological motors to engineer systems for nanoscale transport and assembly. Nature Nanotechnology, 2008. 3(8): 465-475.

[4] I.F. Akyildiz, F. Brunetti, and C. Blázquez, Nanonetworks: A new communication paradigm. Computer Networks, 2008. 52(12): 2260-2279.

[5] T. Nakano, T. Suda, M. Moore, et al., Molecular communication for nanomachines using intercellular calcium signaling. Proceedings of 2005 th IEEE Conference on Nanotechnology. Nagoya, Japan, Jul. 1115, 2005(2): 478-481.

[6] A. Enomoto, M. Moore, T. Nakano, et al., A molecular communication system using a network of cytoskeletal filaments. Technical Proceedings of the 2006 NSTI Nanotechnology Conference and Trade Show, Volume 1. Boston, USA., May 7-11, 2006: 725-728.

[7] Y. Moritani, S. Hiyama, and T. Suda. Molecular communication among nanomachines using vesicles. Technical Proceedings of the 2006 NSTI Nanotechnology Conference and Trade Show, Volume 2. Boston, USA., May 7-11, 2006: 705-708.

[8] S. Hiyama, Y. Moritani, T. Suda, et al., An autonomous molecular transport system using DNAs and motor proteins in molecular communication. Proceedings of the 2nd International ICST Conference on Bio-Inspired
Models of Network, Information, and Computing Systems, BIONETICS 2007. Budapest, Hungary, Dec. 10-13, 2007: 135-138.

[9] A.W. Eckford, N. Farsad, S. Hiyama, et al., Microchannel molecular communication with nanoscale carriers: Brownian motion versus active transport. Proceedings of 10th IEEE International Conference on Nanotechnology. Aug. 17-20, 2010: 2010: 854-858.

[10] M. Pierobon, I.F. Akyildiz, A physical end-to-end model for molecular communication in nanonetworks. IEEE Journal on Selected Areas in Communications, 2010. 28(4): 602-611.

[11] A.W. Eckford, Achievable information rates for molecular communication with distinct molecules. Proceedings of $20072^{\text {nd }}$ Bio-Inspired Models of Network, Information and Computing Systems. Budapest, Hungary, Dec. 10-13, 2007: 313-315.

[12] M. Pierobon, I.F. Akyildiz, Capacity of a diffusion-based molecular communication system with channel memory and molecular noise. IEEE Transactions on Information Theory, 2013. 59(2): 942-954.

[13] K. Srinivas, A.W. Eckford, and R.S. Adve, Molecular communication in fluid media: The additive inverse gaussian noise channel. IEEE Transactions on Information Theory, 2012. 58(7): 4678-4692.

[14] A.C. Heren, H.B. Yilmaz, C.-B. Chae, et al., Effect of degradation in molecular communication: Impairment or enhancement? IEEE Transactions on Molecular, Biological and Multi-Scale Communications, 2015. 1(2): 217-229.

[15] C.T. Chou, Impact of receiver reaction mechanisms on the performance of molecular communication networks. IEEE Transactions on Nanotechnology, 2015. 14(2): 304317.

[16] T. Nakano, M.J. Moore, F. Wei, et al., Molecular communication and networking: opportunities and challenges. IEEE Transactions on Nanobioscience, 2012. 11(2):135-148.

[17] N. Farsad, H.B. Yilmaz, A. Eckford, et al., A Comprehensive Survey of Recent Advancements in Molecular Communication. IEEE Communications Surveys and Tutorials, 2016. 18(3):1887-1919.

[18] T. Nakano, T. Suda, Y. Okaie, et al., Molecular communication among biological nanomachines: A layered architecture and research issues. IEEE Transactions on Nanobioscience, 2014. 13(3):169-197.

[19] W. Guo, T. Asyhari, N. Farsad, et al., Molecular communications: Channel model and physical layer techniques. IEEE Wireless Communications, 2016. 23(4): 120-127.

[20] R.V. Sternberg, DNA codes and information: Formal structures and relational causes. Acta Biotheoretica, 2008. 56(3): 205-232.

[21] R.W. Harrison, I.V. Kourinov, and I.T. Weber, Modeling protein-ligand interactions. Biophysical Journal, 1995. 68(2 PART 2): A6.

[22] D. Kilinc, O.B. Akan, Receiver Design for Molecular Communication. IEEE Journal on Selected Areas in Communications, 2013. 31(12): 705-714.

[23] R. Mosayebi, H. Arjmandi, A. Gohari, et al., Diffusion based molecular communication: A simple near optimal receiver. Proceedings of 2014 Iran Workshop on Communication and Information Theory. Tehran, Iran, May 7-8, 2014: 1-4.

[24] T.E. Mallouk, A. Sen, Powering Nanorobots. Scientific American, 2009. 300(5):72-77.

[25] Y.Z. Du, Y. Hiratsuka, S. Taira, et al., Motor protein nanobiomachine powered by self-supplying ATP. Chemical Communications, 2005(16): 2080-2082.

[26] W. Gao, S. Sattayasamitsathit, K.M. Manesh, et al., 
Magnetically powered flexible metal nanowire motors. Journal of the American Chemical Society, 2010. 132(41): 14403-14405.

[27] S. Sanchez, A.A. Solovev, S.M. Harazim, et al., Microbots swimming in the flowing streams of microfluidic channels. Journal of the American Chemical Society, 2011. 133(4): 701-703.

[28] I.F. Akyildiz, F. Brunetti, and C. Blazquez, Nanonetworks: A new communication paradigm. Computer Networks, 2008. 52(12): 2260-2279.

[29] Y. Moritani, S. Hiyama, and T. Suda, Molecular communication for health care applications. Proceedings. Proceedings of Fourth Annual IEEE International Conference on Pervasive Computing and Communications Workshop-PerCom Workshop 2006. Pisa, Italy, Mar. 1317, 2006: 549-553.

[30] M. Hirabayashi, A. Nishikawa, F. Tanaka, et al., Design of molecular-based network robots - Toward the environmental control. Proceedings of $201111^{\text {th }}$ IEEE International Conference on Nanotechnology. Aug. 15-18, 2011: 313-318.

[31] B. Atakan, O.B. Akan, and S. Balasubramaniam, Body area nanonetworks with molecular communications in nanomedicine. IEEE Communications Magazine, 2012. 50(1): 28-34.

[32] O. Veiseh, J.W. Gunn, and M.Q. Zhang, Design and fabrication of magnetic nanoparticles for targeted drug delivery and imaging. Advanced Drug Delivery Reviews, 2010. 62(3): 284-304.

[33] H. Craighead, Future lab-on-a-chip technologies for interrogating individual molecules. Nature, 2006. 442(7101): 387-393

[34] T. Nakano, Biologically Inspired network systems: A review and future prospects. IEEE Transactions on Systems Man and Cybernetics Part C-Applications and Reviews, 2011. 41(5): 630-643.

[35] M.T. Chen, R. Weiss, Artificial cell-cell communication in yeast Saccharomyces cerevisiae using signaling elements from Arabidopsis thaliana. Nature Biotechnology, 2005. 23(12): 1551-1555.

[36] S. Basu, Y. Gerchman, C.H. Collins, et al., A synthetic multicellular system for programmed pattern formation. Nature, 2005. 434(7037): 1130-1134.

[37] L.C. You, R.S. Cox, R. Weiss, et al., Programmed population control by cell-cell communication and regulated killing. Nature, 2004. 428(6985): 868-871.

[38] A. Guney, B. Atakan, and O.B. Akan, Mobile ad hoc nanonetworks with collision-based molecular communication. IEEE Transactions on Mobile Computing, 2012. 11(3): 353-366.

[39] A. Einolghozati, M. Sardari, F. Fekri, et al., Collective sensing-capacity of bacteria populations. Proceedings of 2012 IEEE International Symposium on Information Theory Proceedings. Cambridge, USA., Jul. 1-6, 2012: $2959-2963$.

[40] Y. Sasaki, Y. Shioyama, W.-J. Tian, et al., A nanosensory device fabricated on a liposome for detection of chemical signals. Biotechnology and Bioengineering, 2010. 105(1): $37-43$.

[41] M. Mukai, K. Maruo, J.I. Kikuchi, et al., Propagation and amplification of molecular information using a photoresponsive molecular switch. Supramolecular Chemistry, 2009. 21(3-4): 284-291.

[42] D.A. LaVan, T. McGuire, and R. Langer, Small-scale systems for in vivo drug delivery. Nature Biotechnology, 2003. 21(10): 1184-1191.

[43] T. Suda, M. Moore, T. Nakano, et al., Exploratory research on molecular communication between nanomachines. Proceedings of Genetic and Evolutionary Computation Conference. Washington, D.C., USA., Jun. 25-29, 2005.
[44] T. Nakano, Y.-H. Hsu, W.C. Tang, et al., Microplatform for intercellular communication. 2008 3rd IEEE International Conference on Nano/Micro Engineered and Molecular Systems. Sanya, China, Jan. 6-9, 2008: 476479.

[45] T. Nakano, T. Koujin, T. Suda, et al., A locally-induced increase in intracellular $\mathrm{Ca} 2+$ propagates cell-to-cell in the presence of plasma membrane $\mathrm{Ca} 2+$ ATPase inhibitors in non-excitable cells. Febs Letters, 2009. 583(22): 35933599.

[46] M. Gregori, I.F. Akyildiz, A new nanonetwork architecture using flagellated bacteria and catalytic nanomotors. IEEE Journal on Selected Areas in Communications, 2010. 28(4): 612-619.

[47] A.A. Solovev, W. Xi, D.H. Gracias, et al., Self-propelled nanotools. Acs Nano, 2012. 6(2): 1751-1756.

[48] M.S. Kuran, H.B. Yilmaz, and T. Tugcu, A tunnel-based approach for signal shaping in molecular communication. Proceedings of 2013 IEEE International Conference on Communications Workshops. 2013. 776-781.

[49] H.C. Berg, Random walks in biology. Princeton University Press, 1993.

[50] M.Ş. Kuran, H.B. Yilmaz, and T. Tugcu. A tunnel-based approach for signal shaping in molecular communication. Proceedings of 2013 IEEE International Conference on Communications Workshops. Budapest, Hungary, Jun. 9-13, 2013: 776-781.

[51] N.-R. Kim, A.W. Eckford, and C.-B. Chae, Symbol interval optimization for molecular communication with drift. IEEE Transactions on Nanobioscience, 2014. 13(3): 223-229.

[52] S. Kadloor, R.S. Adve, and A.W. Eckford, Molecular communication using brownian motion with drift. IEEE Transactions on Nanobioscience, 2012. 11(2): 89-99.

[53] B. Alberts, D. Bray, J. Lewis, et al., Molecular Biology of the Cell (3rd edn). Trends in Biochemical Sciences, 1995. 20(5): 210-210.

[54] Y. Okaie, T. Nakano, T. Hara, et al., Cooperative target tracking by a mobile bionanosensor network. IEEE Transactions on Nanobioscience, 2014. 13(3): 267-277.

[55] M.J. Moore, T. Suda, and K. Oiwa, Molecular communication: Modeling noise effects on information rate. IEEE Transactions on Nanobioscience, 2009. 8(2): $169-180$

[56] N. Farsad, A.W. Eckford, S. Hiyama, et al., A simple mathematical model for information rate of active transport molecular communication. Proceedings of 2011 IEEE Conference on Computer Communications Workshops. Apr. 10-15, 2011: 473-478.

[57] T. Nakano, J.-Q. Liu, Design and analysis of molecular relay channels: An information theoretic approach. IEEE Transactions on Nanobioscience, 2010. 9(3): 213-221.

[58] A.C. Heren, M.Ş. Kuran, H.B. Yilmaz, et al., Channel capacity of calcium signalling based on inter-cellular calcium waves in astrocytes. Proceedings of 2013 IEEE International Conference on Communications Workshops. Budapest, Hungary, Jun. 9-13, 2013: 792 - 797.

[59] N. Farsad, W. Guo, and A.W. Eckford, Tabletop molecular communication: Text messages through chemical signals. PLOS ONE, 2013, 8(12): e82935.

[60] L. Lin, C. Yang, J. Wang, et al., Evaluation of digital baseband modulation schemes for molecular communication in nanonetworks. Proceedings of 2014 Sixth International Conference on Ubiquitous and Future Networks. Shanghai, China, 2014: 297 - 302.

[61] L. Lin, C. Yang, M. Ma, et al., A clock synchronization method for molecular nanomachines in bionanosensor networks. IEEE Sensors Journal, 2016. 16(19): 71947203.

[62] L. Lin, C. Yang, M. Ma, et al., Diffusion-based clock 
synchronization for molecular communication under inverse Gaussian distribution. IEEE Sensors Journal, 2015. 15(9): 4866-4874.

[63] Z. Luo, L. Lin, and M. Ma. Offset estimation for clock synchronization in mobile molecular communication system. Proceedings of 2016 IEEE Wireless Communications and Networking Conference. Doha, Qatar, Apr. 3-6, 2016: 1-6.

[64] L. Lin, C. Yang, S. Ma, et al., Parameter estimation of inverse Gaussian channel for diffusion-based molecular communication. Proceedings of 2016 IEEE Wireless Communications and Networking Conference. Doha, Qatar, Apr. 3-6, 2016: 1 - 6

[65] A. Noel, K.C. Cheung, and R. Schober, Joint channel parameter estimation via diffusive molecular communication. IEEE Transactions on Molecular, Biological, and Multi-Scale Communications, 2015. 1(1): 94-107.

[66] B. Tepekule, A.E. Pusane, H.B. Yilmaz, et al., ISI mitigation techniques in molecular communication. IEEE Transactions on Molecular, Biological, and Multi-Scale Communications, 2015. 1(2): 202-216.

Copyright $(2016$ Hao Yan, Ge Chang, Tianhao Sun, Yingzhan $\mathrm{Xu}$, Zhongke Ma, Tao Zhou, and Lin Lin. This is an open-access article distributed under the terms of the Creative Commons Attribution License, which permits unrestricted use, distribution, and reproduction in any medium, provided the original author and source are credited. 